\title{
Sucesso e falha de extubação em recém-nascidos prematuros até 32 semanas de idade gestacional
}

\author{
Success and failure of extubation in premature \\ newborns up to 32 weeks of gestational age
}

\section{Rafael Jurkevicz ${ }^{1}$ (1) \\ Marimar Goretti Andreazza² (1) Évellin Oliveira Gomes ${ }^{3}$ (1) Ana Lúcia Sarquis Oliveira ${ }^{4}$ (1) Rubneide Barreto Silva Gallo ${ }^{5}$ (1)}

1,2,4,5Universidade Federal do Paraná (Curitiba). Paraná, Brasil. rafaeljurkevicz@hotmail.com, marimar.andreazza@gmail.com, anasarquis@terra.com.br, rubneidegallo@gmail.com ${ }^{3}$ Autora para correspondência. Universidade Federal do Paraná (Curitiba). Paraná, Brasil. evellin.gomes@hc.ufpr.br

RESUMO | INTRODUÇÃO: Os benefícios da ventilação mecânica em neonatos prematuros se complementam com o sucesso na sua retirada. OBJETIVO: Avaliar os parâmetros ventilatórios e gasométricos pré extubação e identificar possíveis fatores que possam contribuir na decisão da extubação endotraqueal em recém-nascidos prematuros até 32 semanas. MÉTODOS: Tratase de um estudo prospectivo, de caráter observacional. A amostra do estudo foi selecionada de forma não probabilística. Foram incluídos prematuros com idade gestacional até 32 semanas, e em ventilação mecânica invasiva por no mínimo 24 horas. E os critérios de exclusão foram recém-nascidos com malformações, cardiopatias e os transferidos para outros hospitais antes da primeira extubação eletiva. Os dados sobre o diagnóstico clínico, dados vitais, parâmetros da ventilação mecânica e gasometria arterial, registrado em uma ficha elaborada pelos pesquisadores e coletados diariamente, uma vez por dia desde o primeiro dia de ventilação mecânica invasiva até o momento de retirada do tubo endotraqueal, sendo o recém-nascido acompanhado até 7 dias após a extubação. O desmame e a extubação seguiu o protocolo da Unidade de Terapia Intensiva Neonatal (UTIN) (presença de respiração espontânea regular e com reflexo de tosse, $\mathrm{FiO} 2<40 \%$ para manter saturação 90\%; pressão inspiratória entre $15-18 \mathrm{cmH2O}$; FR=15 a 20 ipm; pH > 7,25 mmHg e PaCO2 < 50 mmHg). RESULTADOS: Dos 20 recém-nascidos prematuros incluídos no estudo, 14 eram do sexo masculino, a média de idade gestacional foi de $28,9 \pm 2,12$ semanas e a média do peso ao nascimento foi $1069,5 \mathrm{~g} \pm 375,5$. Em $75 \%$ dos casos, a intubação foi devido à síndrome do desconforto respiratório neonatal. Do total da amostra, 40\% apresentaram. Não houve diferença significativa entre os grupos sucesso e falha na extubação, quanto aos dados ventilatórios e gasométricos analisados pré e pós extubação $(p>0,05)$. Na regressão logística, observou-se que a fração inspirada de oxigênio $(p=0,03)$ e a pressão média de vias aéreas ( $p=$ 0,03 ) apresentaram significância como preditor para extubação. O tempo de uso da ventilação mecânica invasiva não apresentou significância estatística $(p=0,06)$, para o desfecho avaliado. CONCLUSÃo: Neste estudo, os parâmetros mínimos ventilatórios como FiO2 e MAP se relacionam diretamente com o sucesso da extubação, bem como estar atento às condições clínicas do paciente auxiliam a equipe a nortear o desmame e programar uma extubação mais criteriosa e segura.

PALAVRAS-CHAVE: Recém-nascido prematuro. Extubação. Ventilação mecânica. Unidades de terapia intensiva neonatal. Nascimento prematuro. Falha na extubação.
ABSTRACT | INTRODUCTION: The benefits of mechanical ventilation in preterm infants are complemented by successful extraction. OBJECTIVE: To evaluate ventilatory and gasometric parameters before extubation and to identify possible factors that may contribute to the endotracheal extubation decision in preterm infants up to 32 weeks. METHODS: This is a prospective observational study. The study sample was selected in a non-probabilistic way. Preterm infants with a gestational age of up to 32 weeks and invasive mechanical ventilation for at least 24 hours were included. Exclusion criteria were newborns with malformations, heart disease, and those transferred to other hospitals before the first elective extubation. Weaning and extubation followed the protocol of the Neonatal Intensive Care Unit (NICU) (presence of regular spontaneous breathing with a cough reflex, $\mathrm{FiO} 2<40 \%$ to maintain $90 \%$ saturation; inspiratory pressure between $15-18 \mathrm{cmH} 2 \mathrm{O}$; RR $=15$ to $20 \mathrm{ipm}$; $\mathrm{pH}>7.25 \mathrm{mmHg}$ and $\mathrm{PaCO} 2<50 \mathrm{mmHg}$ ). The newborn was followed up to 7 days after extubation. RESULTS: 20 preterm newborns were included, in 75\% of the cases intubation was due to neonatal respiratory distress syndrome. Of the total sample, $40 \%$ were unsuccessful. In the logistic regression, it was observed that the inspired oxygen fraction $(p=0.03)$ and the mean airway pressure $(p=0.03$ ) showed significance as a predictor of extubation. The time of use of invasive mechanical ventilation was not statistically significant ( $p=0.06$ ) for the evaluated result. CONCLUSION: In this study, ventilatory and blood gas parameters did not influence the success or failure outcome. However, these data associated with the patient's condition can help guide and schedule safer extubation.

KEYWORDS: Infant, premature. Extubation. Mechanical ventilation. Intensive care units, neonatal. Premature birth. Extubation failure. 


\section{Introdução}

A prematuridade é considerada, atualmente, um problema de saúde pública, sendo um dos principais fatores de risco para morbidade e mortalidade neonatal1 ${ }^{1}$ Segundo a Organização Mundial de Saúde (OMS), é considerado recém-nascido prematuro aquele bebê que nasce antes de completar 37 semanas de gestação², podendo ser classificado de acordo com a idade gestacional em prematuro extremo $(<28$ semanas), prematuro moderado ( 28 a $31+6$ semanas) e prematuro tardio (32 a $36+6$ semanas) ${ }^{3}$.

Uma das principais complicações que acomete o recém-nascido prematuro é a síndrome do desconforto respiratório, que é um distúrbio causado por imaturidade pulmonar e pequena quantidade de surfactante, que compromete a integridade alveolar, dificultando as trocas gasosas normais. A síndrome do desconforto respiratório neonatal ocorre no momento ou logo após o nascimento, antes de 24 horas de vida e aumenta em gravidade durante as primeiras 48 horas de vida ${ }^{4}$, e é a causa mais comum de internação em Unidade de Terapia Intensiva Neonatal (UTIN) ${ }^{5}$. A incidência de síndrome do desconforto respiratório neonatal é maior conforme diminui a idade gestacional $1,6,6$, variando entre $21,3 \%$ a $56,9 \%$, conforme a literatura $\mathrm{z}^{\not \underline{8}}$.

Dessa forma, é comum o recém-nascido prematuro principalmente o prematuro extremo, necessitar de suporte ventilatório invasivo para manter adequada ventilação e oxigenação após o nascimentọ; cerca de $89 \%$ dos recém-nascidos prematuros de extremo baixo peso são ventilados nos primeiros dias de vida ${ }^{10}$. Entretanto, o uso prolongado do suporte ventilatório invasivo bem como parâmetros mal ajustados pode provocar lesões pulmonares como pneumotórax, enfisema pulmonar intersticial e displasia broncopulmonar1112, sendo esta última a principal complicação do uso prolongado da ventilação mecânica invasiva.

Sendo assim, um dos desafios para a equipe multiprofissional é reduzir o tempo de ventilação mecânica invasiva, prevenindo tanto as complicações relacionadas ao uso da ventilação mecânica, quanto a falha de extubação e reintubação ${ }^{13}$. As intervenções que diminuem a exposição à ventilação mecânica invasiva e resultam em extubação bem-sucedida apresentam benefícios a longo prazo ${ }^{14}$. A extubação bem-sucedida é definida como a não necessidade de reintubação durante uma janela de tempo pré determinada, porém em prematuros essa janela de observação pode variar de 12 horas a sete dias ${ }^{14}$.

A hipótese que norteou a pesquisa foi de que os parâmetros ventilatórios utilizados na ventilação mecânica influenciam no desmame precoce e no sucesso da extubação. Dessa forma, o objetivo deste estudo foi avaliar os parâmetros ventilatórios e gasométricos pré extubação e identificar possíveis fatores que possam contribuir na decisão da extubação endotraqueal em recém-nascidos prematuros que tenham até 32 semanas com idade gestacional.

\section{Métodos}

Trata-se de um estudo prospectivo, de caráter observacional. A pesquisa foi aprovada pelo Comitê de Ética em Pesquisa com seres humanos da instituição onde ocorreu a pesquisa sob o parecer número 2.836 .816 (CAAE 91754318.6.0000.0096). O estudo foi realizado na Unidade de Terapia Intensiva Neonatal - UTIN de um hospital universitário terciário. A coleta de dados ocorreu no período de dezembro/2018 a julho/2019. Os pais assinaram o Termo de Consentimento Livre e Esclarecido para participar do estudo.

Os critérios de inclusão foram recém-nascidos prematuros com idade gestacional até 32 semanas, de ambos os sexos, e em ventilação mecânica invasiva por no mínimo 24 horas. E os critérios de exclusão foram recém-nascidos com malformações, cardiopatias e os transferidos para outros hospitais antes da primeira extubação eletiva. A amostra do estudo foi selecionada de forma não probabilística.

Os dados sobre o diagnóstico clínico, dados vitais, parâmetros da ventilação mecânica e gasometria arterial foram anotados em uma ficha elaborada pelos pesquisadores e coletados diariamente, uma vez por dia, no período da manhã, desde o primeiro dia de ventilação mecânica invasiva até o momento da extubação. O acompanhamento foi realizado por 7 dias após a extubação, sendo considerado para o estudo apenas a primeira extubação eletiva.

Os critérios para desmame e extubação foram definidos de acordo com o protocolo da UTIN estudada, sendo elegível para extubação o recém-nascido com 
presença de respiração espontânea regular e com reflexo de tosse, fração inspirada de oxigênio menor que 40\% para manter saturação 90\%, pressão inspiratória entre $15-18 \mathrm{cmH} 20$, frequência respiratória de 15 a 20 inspirações por minuto, pH menor 7,25 mmHg e PaCO2 menor $50 \mathrm{mmHg}$, que segue as recomendações do Consenso Brasileiro em Ventilação mecânica ${ }^{23}$. A despeito da existência de um protocolo para desmame na UTIN onde este estudo foi realizado, não foi utilizado qualquer teste de prontidão para nortear a extubação dos prematuros, respeitando-se sempre os parâmetros gasométricos e de ventilação citados anteriormente, somados às condições clínicas e hemodinâmicas do recém-nascido, bem como do julgamento clínico da equipe médica.

Sucesso de extubação foi considerado quando o recém-nascido permaneceu 7 dias sem necessidade de reintubação endotraqueal, mantendo adequada saturação e estabilidade clínica (Figura 1).

Figura 1. Fluxograma do desenho do estudo

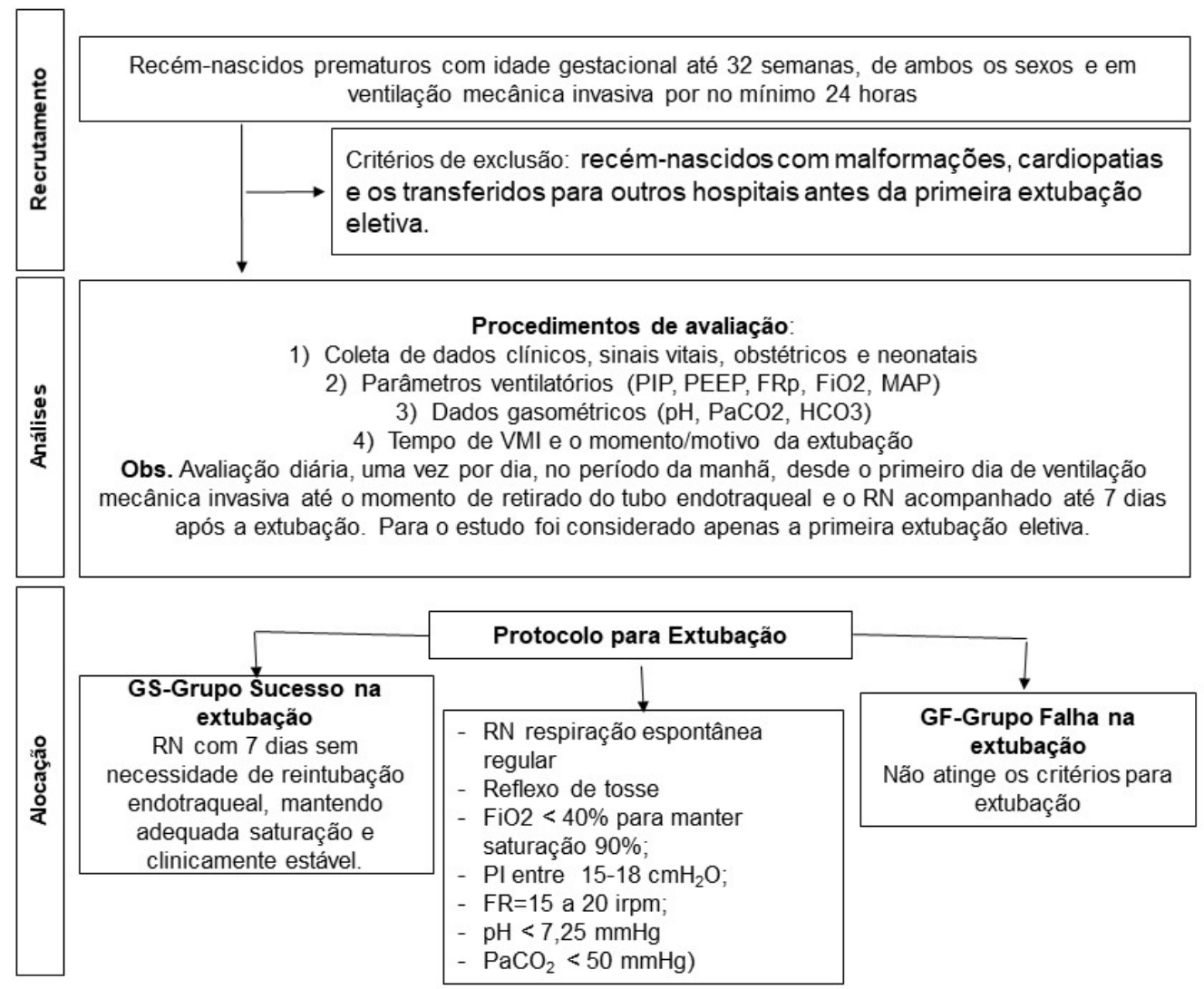


Os dados coletados foram tabulados em uma planilha do Microsoft Excel®. A análise dos dados foi realizada utilizando-se técnicas de análise descritiva para a caracterização da amostra utilizando medidas de tendência central e de dispersão (média e desvio padrão) para as variáveis contínuas. Foi utilizado o software estatístico JASP para análise dos dados. Foi utilizado o teste t de Student para comparar as variáveis contínuas nos grupos sucesso ou falha, sendo considerado significativo um valor de $p<0,05$.

Após a análise estatística por meio das médias e desvio padrão ajustou-se um modelo de regressão logística onde os dados foram analisados por meio do Software R 3.6.1 e RStudio 1.2.1335. No modelo de regressão logística, teve-se como variável resposta o desfecho do estudo, neste caso, sucesso ou falha de extubação, para isso utilizou-se os pacotes MASS v. 7.3-51.4 e caret v. 6.0-84, resultando em um modelo final com as variáveis pressão média das vias aéreas e fração inspirada de oxigênio, significativas a $5 \%$ e tempo de ventilação mecânica invasiva significativo a $10 \%$.

\section{Resultados}

Durante o período de coleta de dados, foram admitidos na UTIN 43 recém-nascidos prematuros com até 32 semanas de idade gestacional. Destes, 11 evoluíram com óbito antes da primeira extubação, dois recém-nascidos prematuros foram transferidos para outro hospital antes da primeira extubação eletiva, e 10 recém-nascidos prematuros não necessitaram de suporte ventilatório invasivo durante a internação. A amostra do estudo foi formada por 20 recém-nascidos prematuros até 32 semanas de idade gestacional. Destes, 12 (60\%) tiveram sucesso de extubação e 8 (40\%) apresentam falha de extubação. Os recém-nascidos que falharam foram reintubados em até 144 horas após a extubação.

Dos 20 recém-nascidos prematuros incluídos no estudo, 14 eram do sexo masculino, a média de idade gestacional foi de $28,9 \pm 2,12$ semanas e a média do peso ao nascimento foi $1069,5 \mathrm{~g} \pm 375,5$. Quanto ao escore de apgar no quinto minuto, 6 RN foram classificados com escore entre 4 e 7, e 14 acima de 7. Quando separados por grupo sucesso e falha na extubação, os recém-nascidos apresentaram características semelhantes. As mães de recém nascidos do estudo fizeram uso de corticoide durante o pré-natal. A ventilação mecânica invasiva foi indicada para 15 recém-nascidos prematuros (75\%) devido à síndrome do desconforto respiratório, os dados estão apontados na tabela 1.

Tabela 1. Características gerais dos recém-nascidos de acordo como os grupos sucesso e falha na extubação $n=(20)$

\begin{tabular}{|c|c|c|c|c|c|}
\hline & \multicolumn{2}{|c|}{ Grupo sucesso $(n=12)$} & \multicolumn{2}{|c|}{ Grupo falha $(n=8)$} & \multirow[b]{2}{*}{ Valor $p$} \\
\hline & Média & $\mathrm{DP}$ & Média & DP & \\
\hline IG sem. & 28,83 & $\pm 2,12$ & 28,87 & $\pm 1,45$ & 0,96 \\
\hline IGC sem. & 29,91 & $\pm 2,15$ & 29,62 & $\pm 1,50$ & 0,74 \\
\hline PN (g) & 1094,5 & $\pm 375,5$ & 1031,8 & $\pm 272,6$ & 0,69 \\
\hline $\mathrm{FC}(\mathrm{bpm})$ & 154,6 & $\pm 15,4$ & 150,5 & $\pm 21,24$ & 0,61 \\
\hline FR (irpm) & 49,33 & $\pm 13,98$ & 44,25 & v 13,25 & 0,42 \\
\hline
\end{tabular}

Nota. Student's t-test; IG: idade gestacional; IGC: idade gestacional corrigida no momento da extubação 
Todos os recém-nascidos prematuros após a extubação receberam ventilação não invasiva no modo com pressão positiva intermitente (NIPPV). As causas de falha na extubação no grupo estudado foram: apneia 4 (50\%), complicações gastrointestinais/infecções 2 (25\%), atelectasia 1(12,5\%), e procedimento cirúrgico/exames 1(12,5\%). Ocorreram entre as primeiras 24 horas até 144 horas após a extubação, conforme apresentado na Figura 2.

Figura 2. Gráfico do momento da falha na extubação de acordo com causas

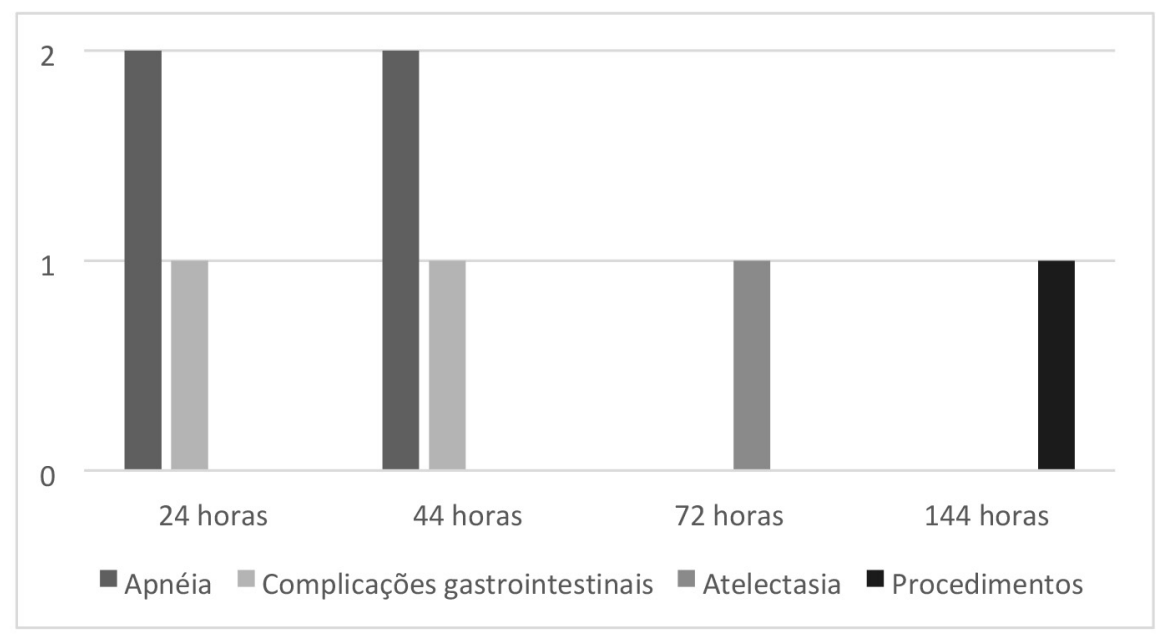

Todos os recém-nascidos prematuros foram extubados seguindo o protocolo de desmame da ventilação mecânica da UTIN do local do estudo. Não houve diferença significativa entre os grupos sucesso e falha na extubação, quanto aos dados gasométricos analisados pré e pós extubação (Tabela 2).

Tabela 2. Dados gasométricos pré e pós extubação dos recém-nascidos dos grupos sucesso e falha na extubação n=(20)

\begin{tabular}{|c|c|c|c|c|c|}
\hline & \multicolumn{2}{|c|}{ Grupo sucesso $(n=12)$} & \multicolumn{2}{|c|}{ Grupo falha $(n=8)$} & \multirow[t]{2}{*}{ Valor $p$} \\
\hline & Média & DP & Média & $\mathrm{DP}$ & \\
\hline pH pré & 7,36 & $\pm 0,07$ & 7,40 & $\pm 0,107$ & 0,34 \\
\hline pH pós & 7,36 & $\pm 0,065$ & 7,32 & $\pm 0,092$ & 0,32 \\
\hline PaCO2 pré & 36,15 & $\pm 6,48$ & 31,50 & $\pm 10,42$ & 0,23 \\
\hline PaCO2 pós & 36,48 & $\pm 6,68$ & 39,98 & $\pm 12,48$ & 0,45 \\
\hline HCO3 pré & 20,70 & $\pm 2,37$ & 21,73 & $\pm 2,03$ & 0,33 \\
\hline HCO3 pós & 20,85 & $\pm 1,54$ & 19,82 & $\pm 1,68$ & 0,19 \\
\hline
\end{tabular}

Nota. Student's t-test. 
De acordo com o modelo de regressão logística foi observado que a pressão média de vias aéreas (MAP) e a fração inspirada de oxigênio (FiO2) foram significativas no desfecho sucesso, indicando que quanto menor esses parâmetros, maiores a chance de sucesso para extubação (Tabela 3). O tempo de VMI foi de 5,9 dias variando entre um e vinte, a média da MAP foi de $7,72( \pm 0,77)$ e a média da FiO2 foi de $0,25( \pm 0,05)$.

Tabela 3. Análise de Regressão Logística das variáveis $n=20$

\begin{tabular}{llll}
\hline & OR & IC 95\% & Valor $p$ \\
\hline Tempo de VMI & 6,33 & $6,15-5,24$ & 0,06 \\
MAP & 1,03 & $3,34-3,17$ & 0,03 \\
FiO2 & 1,86 & $2,98-1,25$ & 0,03 \\
\hline
\end{tabular}

Nota: VMI: ventilação mecânica invasiva; FiO2: fração inspirada de oxigênio; MAP: pressão média de vias aéreas; OR: Odds ratio; IC: Intervalo de Confiança

\section{Discussão}

Devido às particularidades dos recém-nascidos prematuros, diversos autores têm buscado a definição e estabelecimento de critérios objetivos que possam auxiliar no desmame e extubação segura destes recém-nascidos prematuros por meio de testes de prontidão ${ }^{15,16}$. Embora a maioria destes testes apresentam boa sensibilidade para predizer sucesso de extubação, eles possuem baixa especificidade ${ }^{17}$. Sendo a redução dos parâmetros uma prática comum nas UTIN 17,18. Os resultados do estudo estão de acordo com o que a literatura mundial traz, que 0 desmame do paciente neonatal ainda é cercado de dúvidas e desafios.

No presente estudo, a taxa de falha de extubação foi de $40 \%$, em concordância com dados previamente publicados, que apresentam variação entre 23 a 40\%18,19. Portanto, é importante que o desmame e a extubação eletiva sejam bem planejados, prevenindo-se assim os efeitos adversos da ventilação mecânica invasiva prolongada, bem como possíveis complicações relacionadas a reintubação.

A idade gestacional e o baixo peso ao nascimento são variáveis que podem contribuir para o insucesso ou falha na extubação ${ }^{16}$. Neste estudo, não foi observada diferença em relação aos grupos sucesso e falha de extubação em relação ao peso ao nascimento e a idade gestacional.

Em um estudo realizado na França, onde foram avaliados recém-nascidos prematuros abaixo de 32 semanas, observou-se diferenças entre os grupos sucesso e falha de extubação em relação aos parâmetros pH, $\mathrm{PaCO}$, pressão positiva inspiratória e a pressão positiva expiratória ${ }^{21}$; no entanto, neste estudo não foi observado diferença estatisticamente significativa em relação aos parâmetros ventilatórios e gasométricos. Isso possivelmente é justificado pelo uso do protocolo de desmame padronizado da ventilação mecânica invasiva. Embora não tenha sido observada diferença significativa entre os grupos sucesso e falha a literatura mostra que os dados gasométricos como pH, $\mathrm{PaCO} 2$ e HCO3 podem ser utilizados como parâmetros para predizer sucesso de extubação na população neonatal 16,20 .

Durante a ventilação mecânica invasiva, um dos parâmetros controlados é a fração inspirada do oxigênio, que em altas concentrações é lesiva ao recém-nascido. A hiperoxia pode levar à inflamação pulmonar, lesão alveolar, dano pulmonar progressivo e morte ${ }^{22}$. Quando realizada a regressão logística, observou-se que os recém-nascidos prematuros que necessitavam de uma fração inspirada de oxigênio menor para manter adequada saturação periférica de oxigênio durante o desmame da ventilação mecânica invasiva tiveram maior taxa de sucesso na extubação ${ }^{23,24}$. Esses resultados estão em consonância com outros dados já publicados, que demonstraram que a fração inspirada de oxigênio maior que $40 \%$ antes da extubação está associada com maior risco de falha $\$ 23$. 
Outros fatores que apresentaram relação com o desfecho sucesso de extubação foram as variáveis pressão média de vias aéreas e tempo de ventilação mecânica invasiva. O tempo de ventilação mecânica invasiva pode levar à assincronia entre o paciente e o ventilador, bem como períodos prolongados de ventilação mecânica invasiva podem causar lesões nas vias aéreas e estenose subglótica ${ }^{24,25}$.

Todos os recém-nascidos prematuros avaliados neste estudo foram extubados e submetidos a ventilação não invasiva, que tem sido associada com maior taxa de sucesso da extubação, pois auxilia a manutenção das vias aéreas pérvias durante a respiração espontânea ${ }^{26}$. Por ser protocolo do serviço, a modalidade não invasiva de escolha foi a ventilação com pressão positiva intermitente nasal (NIPPV), sendo todos os recém-nascidos extubados colocados nesse modo. Segundo Sant'Anna, esta modalidade pode também ser utilizada quando os recém-nascidos prematuros apresentam falha com o uso do CPAP nasal, na tentativa de prevenir nova reintubação 27 .

Como limitações deste estudo, pode-se citar que só foram utilizados dados da primeira extubação eletiva, não foram consideradas as complicações relacionadas às extubações não planejadas e o pequeno número da amostra; isso provavelmente se justifica pelo limitado tempo para coleta de dados.

\section{Conclusão}

Os parâmetros mínimos ventilatórios como $\mathrm{FiO} 2 \mathrm{e}$ MAP se relacionam diretamente com o sucesso da extubação, bem como estar atento as condições clínicas do paciente auxiliam a equipe a nortear o desmame e programar uma extubação mais criteriosa e segura. Novos estudos são necessários para aprofundamento da temática.

\section{Contribuições dos autores}

Jurkevicz R participou da concepção, delineamento, coleta de dados, interpretação dos dados e redação do artigo cientifico. Andreazza MG participou da concepção, delineamento, interpretação dos dados, análise estatística e redação. Oliveira ALS participou do delineamento e revisão do texto. Gallo R participou do delineamento, interpretação dos dados, analise estatística e redação. Gomes EO participou da concepção, coleta de dados e revisão do artigo.

\section{Conflitos de interesses}

Nenhum conflito financeiro, legal ou político envolvendo terceiros (governo, empresas e fundações privadas, etc.) foi declarado para nenhum aspecto do trabalho submetido (incluindo, mas não se limitando a subvenções e financiamentos, participação em conselho consultivo, desenho de estudo, preparação de manuscrito, análise estatística, etc.).

\section{Referências}

1. Frey HA, Klebanoff MA. The epidemiology, etiology, and costs of preterm birth. Semin Fetal Neonatal Med. 2016;21(2):68-73. https://doi.org/10.1016/j.siny.2015.12.011

2. World Health Organization. Preterm birth [Internet]. Geneva (Suíça): WHO. Disponível em: https://www.who.int/en/news-room/ fact-sheets/detail/preterm-birth

3. Blencowe H, Cousens S, Chou D, Oestergaard M, Say L, Moller A, et al. Born Too Soon: The global epidemiology of 15 million preterm births. Reprod Health. 2013;10(supl 1):S2. https://doi. org/10.1186/1742-4755-10-s1-s2

4. Hiles M, Culpan AM, Watts C, Munyombwe T, Wolstenhulme S. Neonatal respiratory distress syndrome: Chest X-ray or lung ultrasound? A systematic review. Ultrasound. 2017;25(2):80-91. https://doi.org/10.1177/1742271×16689374

5. Wang J, Liu X, Zhu T, Yan C. Analysis of neonatal respiratory distress syndrome among different gestational segments. Int J Clin Exp Med. 2015;8(9):16273-9. Citado em: PMID: 26629144.

6. Dehdashtian M, Malakian A, Aramesh MR, Mazori A, Aletayeb $\mathrm{MH}$, Shirani A, et al. Effectiveness of intratracheal salbutamol in addition to surfactant on the clinical course of newborns with respiratory distress syndrome: a clinical trial. Ital J Pediatr. 2016;42(6):4-8. https://dx.doi.org/10.1186\%2Fs13052-016-0215-1 
7. Souza LL, Santos MBL, Sousa FCA, Silva IS, Araújo FL, Silva IA, et al. Caracterização clínico-epidemiológica dos recém-nascidos em cuidados intensivos. RSD. 2020;9(8):e731986198. https://doi. org/10.33448/rsd-v9i8.6198

8. Ferraresi MF, Arrais AR. Perfil epidemiológico de mães de recém-nascidos admitidos em uma unidade neonatal pública. Rev. Rene [Internet]. 2016;17(6):733-40. Disponível em: http:// www.periodicos.ufc.br/rene/article/view/6453

9. Stoll BJ, Hansen NI, Bell EF, Walsh MC, Carlo WA, Shankaran S, et al. Trends in Care Practices, Morbidity, and Mortality of Extremely Preterm Neonates, 1993-2012. JAMA. 2015;314(10):1039-51. https://doi.org/10.1001/jama.2015.10244

10. Sant'Anna GM, Keszler M. Developing a neonatal unit ventilation protocol for the preterm baby. Early Hum Dev. 2012;88(12):925-9. https://doi.org/10.1016/j. earlhumdev.2012.09.010

11. Reiterer F, Schwaberger B, Freidl T, Schmölzer G, Pichler G, Urlesberger B. Lung-protective ventilatory strategies in intubated preterm neonates with RDS. Paediatr Respir Rev. 2017;23:89-96. https://doi.org/10.1016/j.prrv.2016.10.007

12. Vendettuoli V, Bellù R, Zanini R, Mosca F, Gagliardi L. Changes in ventilator strategies and outcomes in preterm infants. Arch Dis Child Fetal Neonatal Ed. 2014;99(4):F321-4. https://doi. org/10.1136/archdischild-2013-305165

13. Goel N, Chakraborty M, Watkins WJ, Banerjee S. Predicting Extubation Outcomes-A Model Incorporating Heart Rate Characteristics Index.J Pediatr. 2018;195:53-58.e1. https://doi. org/10.1016/j.jpeds.2017.11.037

14. Giaccone A, Jensen E, Davis P, Schmidt B. Definitions of extubation success in very premature infants: a systematic review. Arch Dis Child Fetal Neonatal Ed. 2014;99(2):F124-7. https://doi. org/10.1136/archdischild-2013-304896

15. Kaczmarek J, Kamlin CO, Morley CJ, Davis PG, Sant'anna GM. Variability of respiratory parameters and extubation readiness in ventilated neonates. Arch Dis Child Fetal Neonatal Ed. 2013;98(1):F70-3-4. https://doi.org/10.1136/ fetalneonatal-2011-301340

16. Robles-Rubio CA, Kaczmarek J, Chawla S, Kovacs L, Brown KA, Kearney RE, et al. Automated analysis of respiratory behavior in extremely preterm infants and extubation readiness. Pediatr Pulmonol. 2015;50(5):479-86. https://doi.org/10.1002/ppul.23151

17. Shalish W, Latremouille S, Papenburg J, Sant'Anna GM. Predictors of extubation readiness in preterm infants: a systematic review and meta-analysis. Arch Dis Child Fetal Neonatal Ed. 2019;104(1):F89-F9797. https://doi.org/10.1136/ archdischild-2017-313878
18. Bacci SLLS, Johnston C, Hattori WT, Pereira JM, Azevedo VMGO. Práticas de desmame da ventilação mecânica nas UTIs pediátricas e neonatais brasileiras: Weaning Survey-Brazil. J. bras. pneumol. 2020;46(4):e20190005. https://doi.org/10.36416/1806-3756/ e20190005

19. Chawla S, Natarajan G, Shankaran S, Carper B, Brion LP, Keszler M, et al. Markers of Successful Extubation in Extremely Preterm Infants, and Morbidity After Failed Extubation. J Pediatr. 2017;189(2):113-9. https://doi.org/10.1016/j.jpeds.2017.04.050

20. Wang SH, Liou JY, Chen CY, Chou HC, Hsieh WS, Tsao PN. Risk Factors for Extubation Failure in Extremely Low Birth Weight Infants. Pediatr Neonatol. 2017;58(2):145-50. https://doi. org/10.1016/j.pedneo.2016.01.006

21. Deguines $C$, Bach V, Tourneux P. Facteurs associés à un échec d'extubation chez le nouveau-né prématuré de moins de 32 semaines d'aménorrhée. Arch Pediatr. 2009;16(9):1219-24. https://doi.org/10.1016/j.arcped.2009.05.011

22. Carvalho CG, Procianoy RS, Camargo Neto E, Silveira RC. Preterm neonates with respiratory distress syndrome: Ventilatorinduced lung injury and oxidative stress. J Immunol Res. 2018;2018:6963754. https://doi.org/10.1155/2018/6963754

23. Miyoshi MH. Consenso brasileiro em ventilação mecânica suporte Ventilatório na síndrome do desconforto respiratório do recém-nascido [Internet]. São Paulo; 2015. Disponível em: https:// www.sbp.com.br/fileadmin/user_upload/2015/02/SDR.pdf

24. Cabral LA, Velloso M. Comparação dos efeitos de protocolos de manuseio mínimo em parâmetros fisiológicos de prematuros submetidos à terapia de surfactante exógeno. Brazilian J Phys Ther. 2014;18(2):152-64. https://doi.org/10.1590/S141335552012005000154

25. Vignaux L, Grazioli S, Piquilloud L, Bochaton N, Karam O, Jaecklin $\mathrm{T}$, et al. Optimizing patient-ventilator synchrony during invasive ventilator assist in children and infants remains a difficult task. Pediatr Crit Care Med. 2013;14(7):e316-25. https://doi. org/10.1097/pcc.0b013e31828a8606

26. Weiss B, Kaplan LJ. Oxygen Therapeutics and Mechanical Ventilation Advances. Crit Care Clin. 2017;33(2):293-310. https:// doi.org/10.1016/j.ccc.2016.12.002

27. Shalish W, Sant' Anna GM. Respiratory Care Protocols in Neonatal Intensive Care. In: Aly H, Abdel-Hady H, editores. Respiratory Management of Newborns. Rijeka (Croácia): InTech; 2016. p. 51-69. 